\title{
Músicas, sonidos, ruidos de la ciudad: hacia una polifonía sonora*
}

\author{
Kostas Paparrigopoulos \\ Instituto Tecnológico y Educativo de Creta \\ kpaparrigopoulos@yahoo.com
}

\begin{abstract}
Resumen
En este texto, nos preguntamos por las diferentes bipolarizaciones que se encuentran frecuentemente en la ecología del sonido. La atención estará centrada particularmente en una dicotomía esencial que clasifica los sonidos entre "deseables" e "indeseables", musicales o no musicales, hi-fi o lo-fi, ruido o no ruido. Nos detendremos en algunas observaciones ligadas a esta dicotomía y que conciernen la estética y la libertad en el arte, la formación y la expresión de la subjetividad individual y colectiva, así como las eventuales implicaciones sociales, ecológicas, políticas, incluso geopolíticas.
\end{abstract}

Ruido, subjetivación, ecosofía, ecología sonora, ecología social.

Palabras clave

\section{Musics, sounds and noise of the city: towards a sound polifony}

\begin{abstract}
In this text, we ask for the different bipolarizations that we frequently found in the sound ecology. We will be specially focused on an essential dichotomy which classifies sounds between "desirables" and "undesirables", musicals or non-musicals, hi-fi or lo$\mathrm{fi}$, noise or non-noise. We will dwell on some observations linked to this dichotomy and that concern aesthetics and freedom in art, formation and expression of individual and collective subjectivity, as well as possible social, ecological, political, even geopolitical implications.
\end{abstract}

Keywords

Noise, subjectivation, ecosophy, sound ecology, social ecology.

Musiques, sons e ruídos da cidade: para uma polifonia sonora

Resumo

Neste artigo, perguntamos pelas diferentes bipolaridades frequentemente encontradas na ecologia do som. A atenção será focada principalmente em uma dicotomia essencial que classifica os sons entre "desejável" e "indesejável", musical ou não musical,

* Recibido: 15 de abril de 2017 / Aceptado: 31 de mayo de 2017. 
hi-fi ou lo-fi, ruído ou nenhum ruído. Nos detemos em algumas observações relacionadas com esta dicotomia e relativas à estética e à liberdade na arte, à formação e expressão da subjetividade individual e coletiva, bem como quaisquer implicações geopolíticas - até mesmo políticas ecológicas sociais.

Palavras-chave

Ruído, subjetivação, ecosofia, ecologia do som, ecologia social. 


\section{La música en el siglo XX}

En música, la cuestión de los sonidos deseables o indeseables comporta una dimensión estética y una importancia histórica. Examinando la historia de la música, encontramos asiduamente, en el pasado, obras inspiradas por el ambiente sonoro de la naturaleza; esta última, con frecuencia, apolínea, apacible e idílica, pero también, a veces, dionisiaca, agitada, orgiástica. Podemos citar ejemplos bien conocidos, como el canto de los pájaros o los ruidos de tormentas en Antonio Vivaldi, las olas del mar en Claude Debussy o el despertar de la naturaleza en Gustav Mahler y Arnold Schönberg.

Sin embargo, desde el comienzo del siglo XX, los compositores se interesaron, asimismo, en los sonidos producidos por la actividad humana. Los ruidos de la ciudad moderna, especialmente los que son emitidos por máquinas, atraen la atención de los compositores, en particular de aquellos llamados de vanguardia. Estos compositores se sienten fascinados por el progreso y las promesas de la revolución industrial, pero también y, por sobre todo, por el universo sonoro que acompaña esta revolución. Se podría así decir que, si en el curso de los siglos pasados hubo una "emancipación de la disonancia", a partir del siglo XX fue el turno de la "emancipación del ruido": en Luigi Russolo, el despertar de la ciudad no es más idílico, sino lleno de ruidos mecánicos. Arthur Honegger se fascinaba con los trenes, en particular, con los más potentes $-y$ sin duda los más ruidosos. Por su parte, Edgar Varèse introduce el "ruido" de las percusiones de sirenas mecánicas en las salas de concierto.

En la segunda mitad del siglo XX, la tendencia va a continuar y será incluso amplificada. Pierre Schaeffer y los compositores de la "música concreta" van a utilizar en sus trabajos únicamente sonidos grabados. El magnetófono permite el registro, la edición y la difusión de prácticamente todos los sonidos del ambiente, ruidosos o no. Al mismo tiempo, la música electrónica permitirá enriquecer nuestro universo sonoro con nuevos sonidos inimaginables, inesperados hasta entonces. Del otro lado del Atlántico, John Cage, el admirador de los sonidos de la vida cotidiana, llega al punto de encontrar más interesantes los sonidos del tráfico de Nueva York que la música de Beethoven o Mozart. Para lannis Xenakis, los ruidos de la multitud durante las manifestaciones políticas o los estallidos de las metralletas en las calles de Atenas serán una fuente de inspiración, en sus formalizaciones teóricas y en su música instrumental y electroacústica ${ }^{1}$.

Finalmente, en los años 1960, aparece la música rock gracias también a la comercialización masiva de productos de la revolución electrónica. Se fabrican y se

${ }^{1}$ Para mayor información y un análisis detallado de las relaciones entre música y sonido, consultar Makis Solomos (2013). 
venden a los clientes máquinas musicales (amplificadores, guitarras eléctricas...) a un precio "accesible para todos". Estas máquinas son capaces de modificar el timbre y el volumen sonoro de un instrumento, y, en función de ajustes, también son capaces de hacer bastante ruido. Así, la distorsión de la Stratocaster de Jimmy Hendrix va a dinamizar la estética musical: el ruido se transforma en un medio para liberar la sociedad... El ruido se compromete al servicio de la revolución... Y, por otra parte, las revoluciones siempre han sido más bien ruidosas.

\section{Ecología sonora}

Sin embargo, una nueva aproximación "ecológica" del sonido nace en los años 1960 y 1970, y la relación entre la música, el sonido y el ruido adquiere una nueva dimensión. Todos estos sonidos "fuertes", "agresivos", "dionisiacos" que nutrieron el modernismo, van a estar dotados de una carga negativa desde el punto de vista de una ecología del sonido. Aquí, la cuestión de los "sonidos indeseables", principalmente los sonidos de una gran intensidad, pero también los sonidos electrónicos, se vuelve preponderante. En los años 1970, Murray Schafer introduce el concepto de soundscape (de paisaje sonoro, como es traducido al español), aislando y autonomizando, de alguna manera, la sensación auditiva de las otras sensaciones. Al mismo tiempo, introduce la idea de una dicotomía entre paisaje sonoro de alta-fidelidad ( $h i$-fi) (en los cuales todos los volúmenes sonoros son bajos y equilibrados, pudiendo así escucharse todos los sonidos de un paisaje) y paisaje sonoro de baja fidelidad (lo-fi) (donde los sonidos más fuertes dominan y esconden a los más débiles). Estos conceptos son expresados en términos de la teoría de la información, como relación señal sobre ruido, con el fin de volverlos igualmente medibles en $\mathrm{dB}$, unidad de medida que es, por otra parte, utilizada en la lucha contra la "polución sonora".

Esta dicotomía entre hi-fi y lo-fi es enunciada con una hipótesis moral integrada: el hi-fi es bueno y el lo-fi es malo (Cusack, 2000). El sonido débil, esto es, el silencio, ocupa un lugar eminente en estas estéticas. Por el contrario, el sonido fuerte es considerado indeseable y es casi (o directamente) demonizado. El paisaje sonoro hi-fi es asociado sobre todo al campo pre-industrial, mientras que el lo-fi, al paisaje sonoro resultado de la revolución industrial y post-industrial, particularmente el de las ciudades. Así, el hi-fi devendrá en cierta medida un "ideal" acústico a buscar/alcanzar, bien prometedor, que servirá a la lucha contra la polución sonora. Estas estéticas nos alejan de la ciudad ruidosa y nos llevan a la naturaleza apacible. Los sonidos fuertes de la ciudad son cuestionados y confrontados a los sonidos del campo y, en muchos casos, se intentará transportar el paradigma del campo a la ciudad. Se diría aquí, casi, que la ciudad "ideal" debería parecerse (al menos acústicamente) al campo. 
Definiendo el paisaje sonoro, Murray Schafer abre al mismo tiempo la vía para las nuevas artes sonoras. En su famoso libro The Soundscape: Our Sonic Environment and The Tuning of the World, escribía que el término paisaje sonoro "puede referirse tanto a ambientes reales como a construcciones abstractas, como las composiciones o montajes sobre banda, en particular cuando son consideradas como haciendo parte del ambiente" (Schafer, 1993: 274-275). Aparecen así nuevas prácticas sonoras como el field recording y las composiciones de paisajes sonoros (soundscape compositions).

Las composiciones de paisajes sonoros, tal como han sido teorizadas en la Universidad Simon Fraser de Vancouver, cargan también con una moral "ecológica". Según Barry Truax, uno de los pioneros de la ecología acústica, las composiciones de paisajes acústicos deben tener un objetivo: necesitan apuntar a "la reintegración del auditor con el ambiente, en una relación ecológica equilibrada" (Truax, 2008: 106). Sin embargo, atribuyendo un objetivo moral a la composición, se opera al mismo tiempo una alteración importante del rol del compositor, un rol que comienza a confundirse con el del educador. O bien, como más firmemente lo formula François-Bernard Mâche, "[en este caso,] el compositor aparentemente no tiene sino funciones pedagógicas" (2000).

Evidentemente, estas proposiciones de la ecología acústica incitan muchas preguntas: ¿ंes un retorno, más bien nostálgico, hacia el pasado pre-industrial? ¿Una suerte de neoclasicismo, como dirían los historiadores de la música? ¿Un moralismo estético...?

\section{Subjetivación}

Poniendo en práctica la dicotomía entre sonidos deseables y sonidos indeseables, una pregunta, digamos, fundamental se plantea inmediatamente: ¿cómo podemos calificar un sonido como indeseable o como deseable, como ruido o no ruido? ¿Según qué criterios? ¿Biológicos, estéticos, éticos, ecológicos... o incluso políticos o económicos? ¿Se pueden aplicar criterios objetivos, científicos, medibles? ¿O bien criterios subjetivos, culturales y variables según las épocas? ¿Cómo esta misma pregunta encuentra expresiones diferentes según la óptica adoptada?

Una primera observación incumbe a la implicación de la subjetividad individual en el juicio: ¿sonidos deseables o indeseables para quién? Aquí, entramos en el dominio de la riqueza de la subjetividad humana y de las contradicciones que de ahí resultan, cosa que, por otra parte, es señalada por Murray Schafer cuando escribe que: "la música de un hombre puede ser el ruido de otro" (Schafer, 1993: 273). Podríamos detenernos para hacer algunas consideraciones al respecto. 
En tanto enseñantes, nos encontramos con frecuencia ante estudiantes que aman escuchar música rock, house, trance, rap, noise u otras músicas llamadas "ruidosas". Algunos o algunas, entre nosotros o nosotras, hemos sido probablemente amantes de este tipo de músicas cuando teníamos su edad... y algunos, probablemente, siguen todavía escuchándolas. A menudo, este tipo de músicas se escucha poniendo el volumen del canal hi-fi casi hasta el fondo. Evidentemente, mientras más aumenta el volumen del canal hi-fi, más el paisaje sonoro se vuelve lo-fi. A veces, se alcanzan volúmenes en donde se vuelve un problema escuchar al vecino. En estos momentos, los sonidos emitidos por el altoparlante se vuelven la sola y única realidad perceptible. Nos podemos molestar por esta situación, pero no es el caso para todo el mundo. Hay gente que siente un verdadero placer al sumergirse en esta ruidosa "atmósfera" sonora; y se puede remarcar fácilmente que, a mayor edad, más esta forma de placer disminuye.

Lo que es aquí interesante observar, es que la situación en clases puede invertirse cuando es el profesor quien pone fuerte el volumen de una música de un género musical desconocido, o casi, para los estudiantes; por ejemplo, hacer escuchar un extracto de Bohor, de lannis Xenakis (1962), a los estudiantes de primer año. Los estudiantes no se molestan tanto por el volumen sonoro (Xenakis pedía que la pieza fuese ejecutada bien fuerte) como por la presencia de sonoridades poco habituales para sus oídos. Así, una música, un paisaje sonoro o un único sonido pueden volverse indeseables no sólo por su volumen, por sus dB, sino también por su contenido sonoro. Un ejemplo bien conocido es la forma de onda sinusoidal, calificada por la mayoría como un sonido indeseable, incluso a muy bajo volumen.

Otro parámetro susceptible de intervenir y modificar el juicio es el tiempo. ¿Cuándo un sonido es deseable o indeseable? Un mismo sonido puede ser deseable en un momento e indeseable en el otro. La repetitividad temporal de un acontecimiento sonoro puede también cambiar su calificación: un sonido puede ser deseable cuando se le escucha una o dos veces, pero puede tornarse indeseable cuando se repite; por ejemplo, las campanas idílicas de una iglesia en el campo, el primer día de vacaciones, y las mismas campanas luego de una semana...

Otra observación: un sonido puede ser deseable cuando somos nosotros los que lo producimos, cuando se es el emisor del sonido, pero puede volverse indeseable cuando es emitido por otra persona y nosotros devenimos receptores pasivos; por ejemplo, un pianista que trabaja en su casa, y nosotros, que tenemos la "mala fortuna" de habitar el departamento vecino.

Podríamos continuar y encontrar otras situaciones y citar otros ejemplos que indiquen el rol jugado por la subjetividad en la evaluación de un sonido como deseable o indeseable. 


\section{Subjetivación colectiva}

Es evidente que la subjetividad no es solamente individual, sino también colectiva. La subjetividad colectiva de un grupo humano, de una comunidad, integrada por quienes tienen en común ciertas ideas o prácticas, juega igualmente un rol determinante en la evaluación ética y estética de un sonido.

Tomemos el ejemplo de un sonido considerado indeseable en ecología acústica: el sonido "plano". El sonido de un motor, de combustión interna o eléctrico, es un sonido plano típico. Se clasifica en la misma categoría el bordón musical, una nota pedal sobre la cual se apoyan una o más melodías. El sonido plano se caracteriza por una repetición casi interminable; permanece constantemente igual y es calificado generalmente de monótono y aburrido $-y$, en el caso del bordón, incluso de hipnótico. A gran intensidad, se vuelve extremadamente indeseable, digamos, insoportable.

A pesar de la connotación negativa que porta este sonido, existen sonidos planos (e incluso de gran intensidad en $\mathrm{dB}$ ) que son agradables o, al menos, que son calificados de esta manera por poblaciones que han vivido siempre cerca de cascadas de agua, como las que viven cerca de las caídas del Niagara. Para estas poblaciones, una hipotética tentativa política de disminución de dB emitidos por el sonido de las caídas de agua (en nombre de la lucha contra la "polución sonora"), habrá tocado un elemento esencial de su cotidiano, de su identidad y de su singularidad $^{2}$. Se puede decir lo mismo de todos los pueblos del mediterráneo que han vivido, durante siglos, con el canto de las cigarras (que se llama, en efecto, el "ruido de cigarras").

Estas observaciones no significan que debamos ser admiradores del ruido de los motores... aunque hay ciertas personas que encuentran interesante este sonido (y no hablo solamente de los admiradores de carreras de automóviles, sino también de compositores que utilizan estos sonidos en sus composiciones, o de artistas -sonoros o no- que los usan en sus instalaciones u otros también en el cine, etc.). Se trata de subrayar que no se puede enunciar un juicio estético o moral frente a ningún sonido (ni, menos todavía, de aplicar una política sonora), sin tomar en cuenta la singularidad de la subjetividad, individual o colectiva.

De esta manera, parece que la calificación de un sonido como deseable o indeseable rápidamente se convierte en una cosa bastante compleja, dada la cantidad de parámetros que entran en juego. Sobre todo, porque estos parámetros no son exclusivamente sonoros; es decir, no dependen únicamente de la manifestación sonora de un fenómeno físico. Por ende, se vuelve difícil hablar de una "ecología

\footnotetext{
${ }^{2}$ Consultar asimismo la cita de Margaret Fuller en Johnson (2008: 6).
} 
del sonido" sin hablar, al mismo tiempo, de una "ecología" en sentido amplio. En otros términos, la ecología del sonido no puede ser considerada y estudiada como un sistema cerrado, autónomo, sino más bien como un proceso abierto e interdependiente. Evidentemente, pueden estudiarse las características físicas de un fenómeno sonoro o de un paisaje sonoro, como la acústica lo hace ya desde hace siglos; pero, desde el momento en que la sensibilidad humana entra en juego; a partir del momento en donde una relación "ecológica" se establece (en el sentido de una interacción y de una interdependencia entre los diferentes componentes del ambiente), estamos también obligados a considerar el aspecto psíquico y social del hombre.

\section{Dimensión socioecológica}

El relato de la ecología del sonido está sobre todo basado en la idea de que, mejorando la calidad del paisaje sonoro ambiente, se mejora al mismo tiempo la calidad de vida de la sociedad que habita este paisaje sonoro. Es igualmente sobre esta idea que se basan las intervenciones urbanas contra la polución sonora. Sin embargo, puede también formularse una proposición complementaria: al mejorar la calidad de vida de la sociedad (el sistema sociopolítico), se mejora, al mismo tiempo, el paisaje sonoro donde habita esta sociedad.

Me detendré en esta proposición y en ciertas reflexiones que han sido expresadas en relación a la ausencia de análisis sociopolítico en ecología del sonido, análisis que podría abrir nuevas perspectivas, extendiendo así su campo teórico y práctico. A grandes rasgos, el análisis social de la ecología acústica se limita a constar un deterioro del entorno acústico, que comenzó con la revolución industrial y la aparición de máquinas, para desembocar en la revolución electrónica y la esquizofonía ${ }^{3}$. Sin embargo, este análisis no toca las ideologías y las políticas que existen en el fondo (¿incluso en un primer plano?); que definen y orientan el curso del "progreso", dando forma, así, a nuestro ambiente cotidiano -que es igualmente sonoro.

Podemos citar el caso del artista-investigador Gregg Wagstaff, quien propone una ecología del sonido más activa sobre las cuestiones sociales. Estima que "debemos centrarnos más intensamente en los procedimientos y los aparatos políticos (los instrumentos) que reinan en nuestro paisaje sonoro, y no exclusivamente en los sonidos" (Wagstaff, 2004). Califica la aproximación de la ecología acústica schaferiana de "fonocéntrica" y la ecología profunda de Arne Naess de "biocén-

\footnotetext{
${ }^{3}$ La separación de un sonido y su fuente, debido a su grabación sobre un soporte y a su transmisión y reproducción electroacústica (Schafer, 1993: 90).
} 
trica". Por otra parte, constata que, según Andra Mc Cartney, "la ecología del sonido está implícitamente ligada a la ecología profunda". Estima así que la ecología del sonido "haría bien al reflexionar sobre los objetivos de la ecología social, que toma igualmente en cuenta las relaciones sociales y políticas entre la gente". Remarca finalmente, bajo forma de slogan, que "de hecho, el paisaje sonoro está en nuestros oídos, pero no entre nuestras manos" (Waggstaff, 2004).

En el mismo espíritu, propone una conexión de la ecología del sonido con la ecología social de Murray Bookchin. El filósofo norteamericano, uno de los principales representantes de la ecología social, ponía el acento en el vínculo entre el ambiente y la sociedad y, en particular, sobre el hecho de que el primero depende del segundo. Así, escribía que "en último análisis, es imposible realizar la armonización del hombre con la naturaleza sin crear una comunidad humana que viva en equilibrio permanente con su ambiente natural"4 (Bookchin, 1986: 80). Y agregaba que: "lo que confiere literalmente el carácter 'social' a la ecología social, es el reconocimiento del hecho frecuentemente descuidado de que casi todos nuestros problemas ecológicos actuales derivan de problemas sociales profundamente arraigados. Los problemas ecológicos actuales no pueden ser verdaderamente aprehendidos, y menos resueltos, sin hacer claramente frente a los problemas de la sociedad"5 (Bookchin, 1995: 245).

Bookchin se opone al "ambientalismo", que es, según él, una aproximación fragmentaria de los problemas ambientales, "parches" como dice, y lo acusa de no poder dar verdaderas soluciones, manteniendo así un régimen problemático de base (Bookchin, 1987). Es también muy crítico con la "ecología profunda" de Arne Naess, que considera la humanidad como "una mala cosa 'antropocéntrica' -probablemente un producto maligno de la evolución natural- que 'sobrepopulariza' el planeta y 'devora' sus recursos, destruyendo la fauna salvaje y la biosfera" (Bookchin, 1987: 3). Aconseja, así, un cambio profundo de la estructura social con la creación de numerosas pequeñas comunidades ecológicas autónomas y democráticas, que viven en armonía con su ecosistema. Muchas pequeñas comunidades pueden constituir juntas una todavía más grande y así sucesivamente (en Latouche, 2007: 72) (como las muñecas rusas, bromeando sobre su origen ruso).

\footnotetext{
4 "For, in the final analysis, it is impossible to achieve a harmonization of man and nature without creating a human community that lives in a lasting balance with its natural environment".

5 "What literally defines social ecology as 'social' is its recognition of the often overlooked fact that nearly all our present ecological problems arise from deep-seated social problems. Conversely, present ecological problems cannot be clearly understood, much less resolved, without resolutely dealing with problems within society".
} 
Podemos referir también la "ecosofía" de Félix Guattari, proposición parecida, en el sentido de que se anima por las mismas inquietudes ecológicas y el deseo de extender el debate. En su libro Les trois écologies (Las tres ecologías), Guattari (1989) propone una visión tripartita de la ecología: no solamente ambiental, sino también social y mental. Según él, no se puede separar lo ambiental de lo social y lo mental, a riesgo de una deteriorización del conjunto de estos tres dominios. Escribe que "no es justo separar la acción de la mente, del socius y del ambiente. El rechazo a observar de frente la degradación de estos tres dominios, tal como es operada por los medios, confina a una empresa de infantilización de la opinión y de neutralización destructiva de la democracia" (Guattari, 1989: 32).

\section{Dimensión sociocultural}

La última cuestión que abordaré en este texto concierne a una aproximación sociocultural del sonido dotada de características económicas y geopolíticas. Desde 1979, luego de la aparición del libro de Schafer, Mâche escribía: "se diría que Murray Schafer desea a la vez que la Paz Americana controle todos los paisajes sonoros del mundo, siendo su fichaje apenas la primera etapa..." (2000: 213214). Mâche se refería aquí al "World Soundscape Project" ("Proyecto Mundial del Paisaje Sonoro"), un proyecto de registro y reserva en archivo (en esta época en bandas magnéticas) de los paisajes sonoros del mundo, con el fin de preservarlos ante la desaparición?

En su crítica, Mâche hacía alusión a las tentativas de dominación planetaria por las cuales era acusado el imperialismo norteamericano en esta época. Hoy, la dominación se hace cada vez más presente: pasa sobre todo por la economía y está bastante globalizada. En Europa, la crisis reciente, llamada económica, ha hecho aparecer, entre otras cosas, una dicotomía entre el Norte y el Sur. Esta dicotomía, amplificada por los políticos y exagerada por los medios de comunicación masivos, opone los Siemmens y los Volkswagen frente a las cigarras y los $\mathrm{PIGS}^{8}$ del sur. Se trata de una generalización polarizadora que opone también la angelización y el orgullo del fuerte, frente a la demonización y la humillación del débil.

Paralelo a esta dicotomía, y en relación con nuestro tema, se puede observar otra dicotomía que concierne al paisaje sonoro: la que opone al Norte, considerado globalmente silencioso y apacible (como el campo hi-fi), al Sur, considerado

\footnotetext{
${ }^{6}$ Sobre la "ecosofía sonora", consultar Roberto Barbanti (2011).

7 Un proyecto ambicioso, que dio nacimiento a a continuación al "World Forum for Acoustic Ecology" (Foro Mundial por la Ecología Acústica).

${ }^{8}$ Acrónimo en inglés de Portugal, Italia, Grecia, España. El vocablo homófono "pigs" significa cerdo, por lo cual se hace un juego de palabras con las cigarras (n. del t.).
} 
globalmente como ruidoso y agitado (como la ciudad lo-fi). E incluso si este paralelismo parece un poco excesivo, pienso que es interesante detenerse un instante en él. Citaré a Andra McCartney, una figura importante de la ecología del sonido en Canadá, que escribía, refiriéndose a los estudios del paisaje sonoro: "la soledad de los pastizales y de la tierra salvaje es sentimentalizada y opuesta a la familiaridad y la proximidad de lo cotidiano ruidoso de la vida urbana. Al referirnos al paisaje sonoro hi fi como un ejemplo de paisaje sonoro ecológico, ¿̇estamos dando forma a los estudios del paisaje sonoro a través de un enfoque particularmente nórdico y aislacionista? ¿Es lo que queremos?"9 (McCartney, 2010: 24).

En esta referencia, se trata de diferentes realidades sonoras locales que pueden existir en el planeta y, al mismo tiempo, de diferentes disposiciones culturales para la escucha del paisaje sonoro. Se pueden plantear, evidentemente, muchas preguntas al respecto, como: ¿privilegiamos acaso (incluso inconscientemente) ciertos paisajes sonoros familiares y desfavorecemos otros? ¿Tenemos tendencia a generalizar y a transportar (incluso imponer) nuestra propia experiencia cultural local en otro marco, inapropiado? Se puede igualmente formular una pregunta, a riesgo de caer en la trampa de una teoría del complot: ¿es un juego sutil de poder y dominación? ¿Quién puede eventualmente sacar provecho de esta situación?

Permaneciendo en la misma problemática, se pueden efectuar otras consideraciones similares: no se puede no observar, por ejemplo, que el silencio es también una característica del hombre individualizado, aquel que está alejado de la muchedumbre ruidosa. Es también una característica del hombre "civilizado", del hombre "cultivado", que se ubica en contraste contra el salvaje ruidoso e impulsivo. Se puede observar, también, que el silencio es portador de otra simbólica: la de la sumisión. Puede incluso volverse una suerte de castigo; se dice que se hace callar al débil, al agitado, al niño. Imponer el silencio (literal o metafóricamente) es un acto de poder, del mismo modo que la imposición del ruido.

Frente a estas cuestiones, la ecología del sonido toma una posición más bien errónea, de una sociedad sin clases, sin disparidades y sin tensiones entre sus miembros: la imagen de una humanidad homogénea, sin diferencias o singularidades locales. Y crea también la impresión que no hay más que una sola vía, una sola moral que legitima la aplicación de una única realidad social, individual, sonora también, por todas partes en el mundo. Debemos todas y todos volvernos, de alguna manera, idénticos, parecidos, "iguales"... y "calmos".

\footnotetext{
9 "The solitude of the pasture and the wilderness is romanticized and desired in contrast to the familiarity and close quarters of daily, noisy urban life. By referring to the hifi soundscape as an example of an ecological soundscape, are we shaping soundscape studies through a particularly northern and isolationist framework? Is this what we want?".
} 
En efecto, esta tendencia a la homogeneización, que excluye progresivamente las otras voces, no puede tener por efecto sino un empobrecimiento gradual de la diversidad sonora. Y, haciendo un juego de palabras tomado prestado a la música: arriesgamos pasar de una "polifonía" sonora a una "homofonía", esto es una "monofonía" - palabra que hace alusión a la monotonía.

\section{Para concluir}

Bien parece que esta polarización restrictiva entre términos opuestos ( $\mathrm{hi}$-fi/lofi, silencio/ruido...), que excluye la existencia de estados intermedios, no puede ser actualmente superada por una eventual "de-demonización" del ruido. Tal "decolonización del imaginario sonoro" ${ }^{10}$ de los estereotipos establecidos, habría podido también favorecer -por simpatía o analogía- la emergencia de ideas emancipadoras y liberadoras en otros dominios también, de ideas tan necesarias y deseadas actualmente.

\section{Referencias}

\section{Bibliografía}

Barbanti, Roberto (2011). Écologie sonore et technologies du son. Sonorités (6). 9-41.

Bookchin, Murray (1986). Post-Scarcity Anarchism. Montreal: Black Rose Books.

(1987). Social Ecology versus Deep Ecology. Green Perspectives: Newsletter of the Green Program Project, (4-5). 1-23. Recuperado el 10 de septiembre de 2016 de http://dwardmac.pitzer.edu/Anarchist_Archives/bookchin/socecovdeepeco.html

(1995). What is Social Ecology? En James P. Sterba (ed.) Earth Ethics: Environmental Ethics, Animal Rights, and Practical Applications. Toronto: PrenticeHall. 245-259.

Cusack, Peter (2000). Dialogue. Soundscape, the Journal of Acoustic Ecology, (1-2). 8.

Guattari, Félix (1989). Les trois écologies. París: Galilée.

Johnson, Phyllis (2008). "American Frontier Soundscapes: Rehearing the Old West", Soundscape, the Journal of Acoustic Ecology. (8-1). 5-8.

Latouche, Serge (2007). Petit Traité de la Décroissance Sereine. París: Éditions Mille et une nuits.

${ }^{10}$ Según la "decolonización del imaginario económico" de Serge Latouche (2007). 
Mâche, François-Bernard (2000). "Le paysage sonore". Panorama de la musique, (33) 1980. Republicado en: Un demi-siècle de musique... et toujours contemporaine. París: L'Harmattan.

McCartney, Andra (2010). "Ethical questions about working with soundscapes". Soundscape, the Journal of Acoustic Ecology, 10(1). 24-26

Schafer, Raymond Murray (1993). The Soundscape: Our Sonic Environment and The Tuning of the World. Vermont: Destiny Books.

Solomos, Makis (2013). De la musique au son: L'émergence du son dans la musique des xxe et xxie siècles. Rennes: Presses Universitaires de Rennes.

Truax, Barry (2008). Soundscape Composition as Global Music: Electroacoustic Music as Soundscape. Organised Sound, 13(2). 103-109.

Wagstaff, Gregg (2004). Sound, Art \& Environment, Towards a Social-Ecological Soundscape, Thesis, M. Phil, University of East Anglia, Norwich, England. En WFAE Newsletter. Recuperado el 20 de junio de 2008 de http://wfae.proscenia.net/library/newsarchive/2004/07/resources.htm.

\section{Discografía}

Xenakis, Iannis (1962). Bohor (LP). 21 min 30 s. Francia: Salabert.

Traducción de Gustavo Celedón 